\title{
A THEOREM OF THE HAHN-BANACH TYPE FOR LINEAR TRANSFORMATIONS
}

\author{
BY \\ LEOPOLDO NACHBIN( ${ }^{(1)}$
}

Every continuous linear functional defined on a vector subspace of a real normed space can be extended to the whole space so as to remain linear and continuous, and with the same norm $\left({ }^{2}\right)$. The extension of continuous linear transformations between two real normed spaces has been studied by several authors and for a long time it has been recognized that this problem has a close connection with the question of the existence of projections of norm one, and moreover that the nature of the space where the transformations take their values is much more important than that of the space where the transformations have to be defined. It is not known, as far as we can say, what are the precise conditions for the possibility of extending a transformation without disturbing its linearity, continuity, and norm. In this paper we shall give a necessary and sufficient condition, which refers only to the space where the transformation takes its values and does not involve the transformation itself or the space where it is to be defined, for such an extension to be possible: the condition is expressed in terms of a certain "binary intersection property" of the collection of spheres of the normed space (see Theorem 1). After that we proceed to the study of the structure of real normed spaces whose collections of spheres have this property. A first step in this direction is given by the theorem asserting that these normed spaces (provided they contain at least one extreme point in the unity sphere) are simply those that can be made into complete vector lattices with order unity in such a manner that the norm derived from the order relation and the order unity in the natural way is identical to the given norm (see Theorem 2, and Theorem 3 for the finite-dimensional case). In this connection we point out a conjecture which we have not been able to settle, namely that, if the collection of spheres of a normed space has the binary intersection property, then its unity sphere must contain an extreme point. By using some results of S. Kakutani and M. H. Stone, we establish the connection between the normed spaces having the binary intersection property and the spaces of real continuous functions over certain compact Hausdorff spaces (see Theorem 4), or the complete Boolean algebras (see Theorem 6).

Presented to the Society, September 2, 1949; received by the editors February 23, 1949.

(1) Fellow of the U.S.A. State Department.

(2) This is known as the Hahn-Banach theorem: see Banach [2] and Dieudonne [4] where the result is presented as a theorem on convex sets. Numbers in brackets refer to the bibliography at the end of the paper. 
Besides the problem which we consider in this paper, it would be interesting to investigate in the same direction the extension of transformation with preservation of linearity and continuity, but not necessarily of the norm. In this paper we have restricted ourselves to real normed spaces. It is known, as a consequence of the real case, that the Hahn-Banach theorem holds good for normed spaces with the complex numbers or quaternions as (archimedean) scalars. The Hahn-Banach theorem for normed spaces over fields with (nonarchimedean) valuations has also been studied $\left({ }^{3}\right)$. It would be interesting to examine the extension of linear continuous transformation in these more general situations.

The author wishes to acknowledge his indebtedness to Professors A. A. Albert and M. H. Stone, of the University of Chicago, for encouraging him in his work.

Notations and terminology. We shall say that an ordered set is a complete lattice whenever every nonempty subset bounded from above, or from below, has a supremum, or an infimum. An ordered set is said to have the interpolatory property if whenever $x_{1}, x_{2} \leqq y_{1}, y_{2}$ there exists some $z$ such that $x_{1}, x_{2} \leqq z$ $\leqq y_{1}, y_{2}\left({ }^{4}\right)$. By a segment $[a, b]$ in an ordered set we shall mean the set of all $x$ such that $a \leqq x \leqq b$.

All normed spaces to be considered are supposed to be real. The norm $\|T\|$ of a continuous linear transformation $T$ between two normed spaces is defined to be the supremum of $\|T(x)\| /\|x\|$ for all $x \neq 0$ in the domain of $T$. All spheres in normed spaces are supposed to be closed. The unity sphere of a normed space is the sphere of center at the origin and radius unity. By the segment $[a, b]$ in a vector space we mean the set of points of the form $x=\lambda a+(1-\lambda) b$ for $0 \leqq \lambda \leqq 1$. An extreme point of the unity sphere is a point of this sphere which cannot be written as $\lambda a+(1-\lambda) b$, with $0<\lambda<1, a \neq b$ and $a$ and $b$ in the unity sphere.

We notice that the word segment has two distinct meanings. However it will be clear in every case whether we are referring to one or the other notion of segment.

When we write $T: X \rightarrow Y$ we mean a function with $X$ as domain and with values in $Y$. If $T_{1}$ and $T_{2}$ are functions, we write $T_{1}>T_{2}$ to denote that $T_{1}$ is an extension of $T_{2}$, that is, the domain of $T_{1}$ contains that of $T_{2}$ and $T_{1}(x)=T_{2}(x)$ whenever the right-hand side has a meaning. If $T_{1}$ and $T_{2}$ are real functions on the same domain, we write $T_{1} \geqq T_{2}$ to indicate that $T_{1}(x) \geqq T_{2}(x)$ for any $x$.

1. The extension property. Let $\&$ be a given normed space. $\&$ is said to have the extension property if, for any normed space $\mathfrak{X}$ and for any vector subspace $\subseteq$ of $\mathfrak{X}$, every continuous linear transformation $f: \subseteq \rightarrow \mathbb{E}$ has at least one extension $F: \mathfrak{X} \rightarrow \mathbb{E}$ which is linear, continuous, and has the same norm,

(3) See Cohen [3] and the references to A. F. Monna given there.

(4) This notion was introduced by Riesz [12] in a different but equivalent form (see his condition 4, p. 175) in the case of ordered groups. 
that is, $\|F\|=\|f\|$. The Hahn-Banach theorem says simply that every onedimensional normed space has the extension property.

It is clear that every normed space $\mathbb{E}$ with the extension property must be complete. For consider its completion $\mathfrak{X}$, put $\mathfrak{S}=\mathbb{E}$ and call $f: \mathfrak{S} \rightarrow \mathbb{E}$ the identity mapping. Then the continuous linear extension $F: \mathfrak{X} \rightarrow \mathbb{E}$ of $f$ will have $\&$ as its range and will be a continuous mapping of the Hausdorff space $\mathfrak{X}$ into itself which coincides on a dense subset $\mathbb{E}$ of $\mathfrak{X}$ with the identity mapping of $\mathfrak{X}$; therefore $F$ is the identity mapping of $\mathfrak{X}$, and therefore $\mathfrak{X}=\mathfrak{F}$.

In order to state a necessary and sufficient condition for $\&$ to have the extension property, we shall need the following definition. A collection $\mathcal{C}$ of sets will be said to have the binary intersection property if every subcollection of $\mathcal{C}$, any two members of which intersect, has a nonvoid intersection. This binary intersection property belongs to the same family as the compactness property. For each integer $n \geqq 2$ we can define in a natural way the $n$-ary property so as to include the above definition when $n=2$. This more general property is well known in the theory of convex bodies: let us quote only Helly's theorem according to which the collection of compact convex sets in $(n-1)$-dimensional euclidean space has the $n$-ary intersection property.

Our purpose will be to prove the following theorem.

Theorem 1. A normed space has the extension property if and only if the collection of its spheres has the binary intersection property.

Before going into the proof of this result, we shall indicate two well known normed spaces having the binary intersection property. The first example is given by the space of all real bounded functions on a set $\Omega$, considered as a vector space in the usual way, the norm of each function $f$ being the supremum of $|f(x)|$ for $x \in \Omega$. Another example is given by the space of all real bounded measurable functions on a set $\Omega$ endowed with a non-negative completely additive measure such that $\Omega$ can be expressed as a union of countably many measurable sets with finite measure, the vector space operations being defined in the usual way and the norm of each function $f$ being the essential supremum of $|f(x)|$ for $x \in \Omega$. We omit the proof that these two normed spaces have the binary intersection property, because they are complete vector lattices with order unity in the natural way and therefore they are included in a more general situation that we shall consider later (see $\$ 4$ ).

It is not without interest to notice that the extension property is equivalent to the projection property which is defined in the following way. A given normed space $F$ is said to have the projection property if, for any normed space $\mathfrak{X}$ containing $\mathbb{F}$ as a normed subspace, there is a projection of norm one of $\mathfrak{X}$ onto $\mathbb{E}$. One-half of the equivalence is clear from the remark that a projection of $\mathfrak{X}$ onto $\mathfrak{F}$ is simply a linear extension defined on $\mathfrak{X}$ with values in $\mathscr{E}$ of the identity transformation of $₹$. On the other hand it is known that every normed space is isomorphic to a normed subspace of the space of all 
bounded real functions over a certain set: since, as we have already noticed, this space of bounded functions has the extension property, it follows that every normed space $\&$ is a normed subspace of a normed space $E^{\prime}$ with the extension property. If $\&$ is assumed to have the projection property, there exists a projection $p: \mathbb{E} \rightarrow \mathbb{E}$ of $\mathbb{F}^{\prime}$ onto $\mathbb{E}$ with norm unity. Consider then any normed space $\mathfrak{X}$, any vector subspace $\mathfrak{S}$ of $\mathfrak{X}$ and a continuous linear transformation $f: \Im \rightarrow \mathbb{E}$. Then $f$ may also be considered as having its values in $\mathbb{F}^{\prime}$ : therefore it has a continuous linear extension $F^{\prime}: \mathfrak{X} \rightarrow \mathscr{F}^{\prime}$ such that $\left\|F^{\prime}\right\|=\|f\|$. If we put $F=p \circ F^{\prime}$, it is clear that $F: \mathfrak{X} \rightarrow \mathbb{E}$ is a continuous linear extension of $f: \Im \rightarrow \mathbb{E}$ such that $\|F\|=\|f\|$, showing that $\mathbb{E}$ has the extension property.

2. Sufficiency in the main theorem. Consider the normed space $\$$ and assume that its collection of spheres has the binary intersection property. Let $\mathfrak{X}$ be another normed space, $\mathfrak{S}$ a. vector subspace of $\mathfrak{X}$, and $f: \Im \rightarrow \mathbb{E}$ a continuous linear transformation.

Denote by $\Omega$ the collection of all continuous linear transformations $\phi: \mathfrak{B} \rightarrow \mathbb{E}$, where $\mathfrak{B}$ is a vector subspace of $\mathfrak{X}$, such that $\phi>f$ and $\|\phi\|=\|f\|$. Since $f \in \Omega$, we see that $\Omega$ is not empty. Moreover $\Omega$ is a set ordered by the relation $\prec$, and $\Omega$ is inductive, that is, every nonempty totally ordered set $\Gamma \subset \Omega$ has a least upper bound in $\Omega$. In fact, let $\mathfrak{W}$ be the point set union of the domains of all $\phi \in \Gamma$ : since these domains are totally ordered by set inclusion, it follows that $\mathfrak{W}$ is a vector subspace of $\mathfrak{X}$. Define the transformation $\psi: \mathfrak{W} \rightarrow \mathbb{E}$ in the following way: if $x \in \mathfrak{W}$, there exists some $\phi: \mathfrak{B} \rightarrow \mathbb{E}$ belonging to $\Gamma$ such that $x \in \mathfrak{B}$ and then we put $\psi(x)=\phi(x)$. It is not hard to verif $y$ that the transformation so defined is single-valued, linear, continuous, and $\psi>f,\|\psi\|=\|f\|$. Therefore $\psi \in \Omega$ and $\psi$ clearly is the least upper bound of $\Gamma$ in $\Omega$, as desired. Having proved this, we can make use of Zorn's theorem( $\left.{ }^{5}\right)$ and we see that $\Omega$ contains at least a maximal element.

It will then be sufficient to prove that an arbitrary $\phi \in \Omega$ whose domain $\mathfrak{B}$ is not the whole $\mathfrak{X}$ cannot be maximal in $\Omega$. In fact, let $\zeta$ be in $\mathfrak{X}$ but not in $\mathfrak{B}$. Consider the transform $\mathfrak{U}=\phi(\mathfrak{B})$ of $\mathfrak{B}$ under $\phi$. For each $u \in \mathfrak{U}$ define

$$
\rho(u)=k \cdot \inf \|x-\zeta\|
$$

where $x$ runs over the set $\phi^{-1}(u)$ and $k=\|f\|$. If $u_{1}$ and $u_{2}$ are in $\mathfrak{u}$, then

$$
\rho\left(u_{1}\right)+\rho\left(u_{2}\right) \geqq\left\|u_{1}-u_{2}\right\| \text {. }
$$

In fact, if $x_{1} \in \phi^{-1}\left(u_{1}\right)$ and $x_{2} \in \phi^{-1}\left(u_{2}\right)$, then

$$
\left\|x_{1}-\zeta\right\|+\left\|x_{2}-\zeta\right\| \geqq\left\|x_{1}-x_{2}\right\|
$$

together with

$$
\left\|u_{1}-u_{2}\right\|=\left\|\phi\left(x_{1}-x_{2}\right)\right\| \leqq k\left\|x_{1}-x_{2}\right\|
$$

imply

(5) See Lefschetz [10]. 


$$
k\left\|x_{1}-\zeta\right\|+k\left\|x_{2}-\zeta\right\| \geqq\left\|u_{1}-u_{2}\right\|
$$

from which (1) follows. Knowing this, call $S_{u}$ the sphere in the space $\&$ whose center is $u \in \mathfrak{U}$ and whose radius is $\rho(u)$. It is not hard to see that two sp'heres of a normed space intersect if and only if the sum of their radii is not less than the distance of their centers. Therefore relation (1) shows that any two members of the collection $\left\{S_{u}\right\}$ do intersect. By the binary intersection property, there is a point $\xi \in \mathbb{E}$ common to all these spheres, that is,

$$
\|\phi(x)-\xi\| \leqq k\|x-\xi\|
$$

for all $x \in \mathfrak{B}$. Call $\mathfrak{W}$ the vector subspace of $\mathfrak{X}$ spanned by $\mathfrak{B}$ and by $\zeta$. Every element $x \in \mathfrak{W}$ can be uniquely written as $x=v+\lambda \zeta$, where $v \in \mathfrak{B}$ and $\lambda$ is a real number. Define the transformation $\psi: \mathfrak{W} \rightarrow \mathbb{E}$ by $\psi(x)=\phi(v)+\lambda \xi$. It is not hard to verify that $\psi$ is linear. Moreover $\psi$ is continuous and $\|\psi\| \leqq k$, that is,

$$
\|\phi(v)+\lambda \xi\| \leqq k\|v+\lambda \xi\|
$$

for all $v$ and $\lambda$; this is clear if $\lambda=0$, and if $\lambda \neq 0$ it is equivalent to

$$
\left\|\phi\left(-\frac{v}{\lambda}\right)-\xi\right\| \leqq k\left\|\left(-\frac{v}{\lambda}\right)-\zeta\right\|
$$

and therefore follows from (2). On the other hand it is clear that $\psi>\phi$ : this implies $\psi>f$ and also $\|\psi\| \geqq\|f\|=k$. Therefore $\|\psi\|=\|f\|$. We can then assert that $\psi \in \Omega$ and since $\psi \neq \phi$, we see that $\phi$ is not maximal in $\Omega$, as desired.

The above proof differs from Banach's proof, the difference being the fact that a priori we do not have an order relation on $E$ reasonably related to the norm and vector operations (besides the fact that we have used Zorn's theorem, now more popular than transfinite induction). Later, however, we shall see that this difference is not really very strong (see the remark at the end of $\$ 4$ ).

3. Necessity in the main theorem. Consider a normed space $\leftleftarrows$. By an immediate extension of $\mathbb{E}$ we shall mean every normed space $\mathfrak{X}$ containing $\mathbb{E}$ as a normed subspace, such that $\mathscr{F} \neq \mathfrak{X}$ and $\mathbb{E}$ is a maximal vector subspace of $\mathfrak{X}$. Consider some $\zeta$ in $\mathfrak{X}$ but not in $\mathbb{E}$, and define $\rho(x)=\|x-\zeta\|$ for $x \in \mathbb{E}$. We have then a real function on $\mathbb{E}$. The knowledge of this function together with the norm on $\mathbb{E}$ determines the norm on $\mathfrak{X}$. In fact, every element $y \in \mathfrak{X}$ can be written as $y=t+\lambda \zeta$, where $t \in \mathbb{E}$ and $\lambda$ is a real number and then

$$
\|y\|= \begin{cases}\|t\| & \text { if } \lambda=0 \\ |\lambda| \rho(-t / \lambda) & \text { if } \lambda \neq 0 .\end{cases}
$$

The real function $\rho$ possesses the following properties:

(I) $\rho(x)+\rho(y) \geqq\|x-y\|$ for $x, y \in($,

(II) $|\rho(x)-\rho(y)| \leqq\|x-y\|$ for $x, y \in \&$, 
(III) $\rho[\lambda x+(1-\lambda) y] \leqq \lambda \rho(x)+(1-\lambda) \rho(y)$ for $x, y \in($ and $0 \leqq \lambda \leqq 1$, (IV) $\rho(x)>0$ for $x \in($.

Properties (I) and (II) are obvious consequences of the triangle inequality applied to the vertices $x, y$, and $\zeta$. To prove (III) we remark that

$$
\begin{aligned}
\|\lambda x+(1-\lambda) y-\zeta\| & =\|\lambda(x-\zeta)+(1-\lambda)(y-\zeta)\| \\
& \leqq \lambda\|x-\zeta\|+(1-\lambda)\|y-\zeta\| .
\end{aligned}
$$

Finally (IV) means that $x \neq \zeta$. The following lemma shows precisely that the converse of all that has just been stated is true.

LEMMA 1. If $\rho$ is a real function on the normed space $\$$ satisfying the conditions (I), (II), (III), and (IV) above, then there exists an immediate extension $\mathfrak{X}$ of $\mathbb{E}$ and a point $\zeta$ in $\mathfrak{X}$ but not in $\mathbb{F}$ such that $\rho(x)=\|x-\zeta\|$ for $x \in \mathbb{E}$.

To prove the lemma, consider some (not normed) vector space $\mathfrak{X}$ containing $\mathbb{E}$ as a vector subspace such that $\mathbb{E} \neq \mathfrak{X}$ and $\mathbb{E}$ is maximal in $\mathfrak{X}$. Take some $\zeta$ in $\mathfrak{X}$ but not in $\mathfrak{E}$. Every element $y \in \mathfrak{X}$ can be uniquely written as $y=t+\lambda \zeta$, where $t \in \mathbb{E}$ and $\lambda$ is a real number. Define the function $\mu$ on $\mathfrak{X}$ by

$$
\mu(y)= \begin{cases}\|t\| & \text { if } \lambda=0, \\ |\lambda| \rho(-t / \lambda) & \text { if } \lambda \neq 0 .\end{cases}
$$

We shall prove that this is a norm function on $\mathfrak{X}$. It is obvious that $\mu(y) \geqq 0$. By property (IV), if $\lambda \neq 0$, then certainly $\mu(y)>0$. Therefore, if $\mu(y)=0$, then not only $\lambda=0$ but also $\|t\|=0$, that is, $t=0$ and therefore $y=0$. We have $\mu(\alpha y)=|\alpha| \mu(y)$ : if $\alpha=0$ this means simply that $\mu(0)=0$; if $\alpha \neq 0$ and $\lambda=0$, then $\mu(\alpha y)=\|\alpha t\|=|\alpha| \cdot\|t\|=|\alpha| \mu(y)$; and if $\alpha \neq 0$ and $\lambda \neq 0$, then $\mu(\alpha y)=|\alpha \lambda| \rho(-\alpha x / \alpha \lambda)=|\alpha| \mu(y)$. Finally we prove that $\mu$ satisfies the triangle inequality $\mu(y+z) \leqq \mu(y)+\mu(z)$. We find it necessary to divide this part of the proof into the following cases:

(a) $y$ and $z$ belong to $\mathbb{E}$. Then the inequality follows from the triangle inequality on $(5$.

(b) One and only one of the points $y$ and $z$ belongs to $\mathbb{E}$ : for instance $y \notin \notin$ and $z \in \mathbb{E}$. Then we can write $y=t+\lambda \zeta$, with $t \in \mathbb{E}$ and $\lambda \neq 0$, and we have to prove that

$$
|\lambda| \rho\left(-\frac{t+z}{\lambda}\right) \leqq|\lambda| \rho\left(-\frac{t}{\lambda}\right)+\|z\| .
$$

This follows simply from property (II) where we have to replace $x$ and $y$ by $-(t+z) / \lambda$ and $-t / \lambda$, respectively.

Before considering the remaining cases, let us assume that $y, z \notin \notin$ and so $y=t+\lambda \zeta, z=u+\tau \zeta$ with $t, u \in \notin$ and $\lambda, \tau \neq 0$.

(c) $\lambda$ and $\tau$ have the same sign, for instance both are positive. Then we 
have to prove that

$$
(\lambda+\tau) \rho\left(-\frac{t+u}{\lambda+\tau}\right) \leqq \lambda \rho\left(-\frac{t}{\lambda}\right)+\tau \rho\left(-\frac{u}{\tau}\right) .
$$

This follows simply from property (III) with $-t / \lambda,-u / \tau$, and $\lambda /(\lambda+\tau)$ in the places of $x, y$, and $\lambda$, respectively.

(d) $\lambda$ and $\tau$ have different signs and $\lambda+\tau \neq 0$. Then $\lambda+\tau$ has the same sign either of $\lambda$ or of $\tau$. Assume for instance $\lambda>0, \tau<0$, and $\lambda+\tau>0$. Then we have to prove that

$$
(\lambda+\tau) \rho\left(-\frac{t+u}{\lambda+\tau}\right) \leqq \lambda \rho\left(-\frac{t}{\lambda}\right)-\tau \rho\left(-\frac{u}{\tau}\right),
$$

which is equivalent to

$$
(\lambda+\tau) \rho\left(-\frac{t+u}{\lambda+\tau}\right)-(\lambda+\tau) \rho\left(-\frac{t}{\lambda}\right) \leqq-\tau \rho\left(-\frac{t}{\lambda}\right)-\tau \rho\left(-\frac{u}{\tau}\right) .
$$

If we replace $x$ and $y$ by $-(t+u) /(\lambda+\tau)$ and $-t / \lambda$ respectively in property (II), we see that the left-hand side of the above inequality is at most equal to $\|u-\tau t / \lambda\|$. On the other hand, if we replace $x$ and $y$ by $-t / \lambda$ and $-u / \tau$ respectively in property (I), we see that the right-hand side of the above inequality is at least equal to $\|u-\tau t / \lambda\|$. Therefore the inequality is proved.

(e) $\lambda=-\tau$. Assume for instance $\lambda>0$. Then we have to prove that

$$
\|t+u\| \leqq \lambda \rho\left(-\frac{t}{\lambda}\right)+\lambda \rho\left(\frac{u}{\lambda}\right)
$$

This follows simply from property (I) where we have to replace $x$ and $y$ by $-t / \lambda$ and $u / \lambda$, respectively.

We have thus finished the proof that $\mu$ is a norm function on $X$ and now we may use the standard notation $\|y\|$ for $\mu(y)$. From the definition it is clear that $\mathbb{E}$ is then a normed subspace of $\mathfrak{X}$, and so $\mathfrak{X}$ becomes an immediate extension of $\&$ for which $\|x-\zeta\|=\rho(x)$ for $x \in \mathbb{E}$, and the lemma is proved.

For a moment we shall forget about property (IV) and be interested only in properties (I), (II), and (III).

If $\rho$ and $r$ are real functions on $(\xi$, where $\rho$ has properties (I), (II), and (III) and $r \geqq \rho$, then we can say that $r$ has property (I), that is, $r(x)+r(y) \geqq\|x-y\|$. The next lemma shows that the converse is also valid.

LEMMA 2. If $r$ is a real function on a normed space \& such that

$$
r(x)+r(y) \geqq\|x-y\|
$$

for all $x, y \in \notin$, then there exists a real function $\rho$ on $\&$ satisfying (I), (II), and (III) and such that $r \geqq \rho$. 
To establish the above lemma, let us consider the class $\Delta$ of all real functions $f$ on $\mathbb{E}$ satisfying the inequality

$$
f(x)+f(y) \geqq\|x-y\|
$$

for all $x, y \in \mathbb{E}$, so that $r$ is simply a member of this class. If we make $x=y$ in this inequality, we see that $f \geqq 0$ for every $f \in \Delta$. We shall consider $\Delta$ as a set ordered by the relation $f_{1} \leqq f_{2}$. This ordered set is inductive in the sense that every nonempty totally ordered subset $\Gamma \subset \Delta$ has a greatest lower bound. In fact, for each $x \in \mathbb{E}$ call $\phi(x)$ the greatest lower bound of the number $f(x)$ for $f \in \Gamma$. If $x, y \in ほ$ and $\epsilon>0$, there exists some $f_{1} \in \Gamma$ such that $f_{1}(x) \leqq \phi(x)+\epsilon$, and also some $f_{2} \in \Gamma$ for which $f_{2}(y) \leqq \phi(y)+\epsilon$. Since $\Gamma$ is totally ordered, we have either $f_{1} \leqq f_{2}$ or $f_{2} \leqq f_{1}$. Assume the first case. Then $f_{1}(y) \leqq f_{2}(y)$ and so

$$
\|x-y\| \leqq f_{1}(x)+f_{1}(y) \leqq \phi(x)+\phi(y)+2 \epsilon .
$$

Making $\epsilon \rightarrow 0$ we see that $\phi$ satisfies the inequality that shows that $\phi \in \Delta$. It is then clear that $\phi$ is the greatest lower bound of $\Gamma$ in $\Delta$. Having proved this, we can apply Zorn's theorem and conclude the existence of an element $\rho$ minimal in $\Delta$ for which $r \geqq \rho$.

The lemma will then be proved if we show that every $\rho$ minimal in $\Delta$ satisfies conditions (II) and (III) besides condition (I). Consider a number $h \geqq 0$ and a point $x \in \mathcal{F}$, and define the function $\phi$ by

$$
\phi(t)=\left\{\begin{array}{ccc}
h & \text { if } & t=x, \\
\rho(t) & \text { if } & t \neq x .
\end{array}\right.
$$

It is not hard to verify that $\phi \in \Delta$ if and only if

$$
h \geqq \sup \{\|t-x\|-\rho(t)\}
$$

as $t$ runs over $($. Since $\rho$ is minimal we cannot have $\phi \leqq \rho, \phi \neq \rho$ for the function $\phi$ corresponding to such an $h$ and therefore $h \geqq \rho(x)$. Thus every $h \geqq 0$ satisfying (3) is not less than $\rho(x)$. Therefore

$$
0 \vee \sup \{\|t-x\|-\rho(t)\} \geqq \rho(x) \text {. }
$$

Now we remark that

$$
\|x-y\|+\rho(y) \geqq \sup \{\|t-x\|-\rho(t)\}
$$

for any $y \in \mathbb{E}$, that is to say

$$
\rho(t)+\rho(y) \geqq\|t-x\|-\|x-y\|,
$$

for the left-hand side is not less that $\|t-y\|$. From (4) and (5) we get

$$
\|x-y\| \geqq \rho(x)-\rho(y) .
$$

Since $x$ and $y$ are arbitrary, we can interchange $x$ and $y$ and conclude that $\rho$ satisfies condition (II). Consider now two points $x, y \in \mathbb{E}$, a number $\lambda$, 
$0 \leqq \lambda \leqq 1$, and define $z=\lambda x+(1-\lambda) y$. We shall verify that

$$
\lambda \rho(x)+(1-\lambda) \rho(y) \geqq \sup \{\|t-z\|-\rho(t)\} .
$$

In fact, this amounts to

$$
\lambda\{\rho(x)+\rho(t)\}+(1-\lambda)\{\rho(y)+\rho(t)\} \geqq\|t-z\|,
$$

and therefore follows from

$$
\begin{aligned}
\|t-z\| & =\|\lambda(t-x)+(1-\lambda)(t-y)\| \\
& \leqq \lambda\|t-x\|+(1-\lambda)\|t-y\| \\
& \leqq \lambda\{\rho(t)+\rho(x)\}+(1-\lambda)\{\rho(t)+\rho(y)\} .
\end{aligned}
$$

Now if we compare (3) and (6), we see that we can take $h=\lambda \rho(x)+(1-\lambda) \rho(y)$ and conclude $h \geqq \rho(z)$, which means exactly that $\rho$ satisfies condition (III). The lemma is therefore proved.

Having these two lemmas, we can now prove the necessity in Theorem 1.

Consider a normed space $\mathbb{E}$ and assume that there exists a collection $\mathcal{C}_{0}$ of spheres of $\&$ any two of which have a nonempty intersection, but such that $\mathcal{C}_{0}$ has a void intersection. Call $A$ the set of centers of members of this collection. For each $x \in A$ call $r(x)$ the greatest lower bound of the radii of the members of $C_{0}$ with $x$ as center. It is not hard to verify that

$$
r(x)+r(y) \geqq\|x-y\|, \quad x, y \in A .
$$

For each $x \in A$, consider the sphere of center $x$ and radius $r(x)$ and call $\mathcal{C}_{1}$ the collection of spheres obtained in this way. By a remark already used, any two members of $\mathcal{C}_{1}$ intersect, but the collection $\mathcal{C}_{1}$ has a void intersection, for a point common to all members of $\mathcal{C}_{1}$ would be common to all members of $\mathcal{C}_{0}$. Moreover two distinct members of $\mathcal{C}_{1}$ have distinct centers. If $A$ is not the whole space $\xi$, we can enlarge $\mathcal{C}_{1}$ as follows. Let $\xi$ be a fixed point of $A$ and for each $x$ in $\&$ but not in $A$ consider the sphere of center $x$ and radius $\|x-\xi\|+r(\xi)$. Since this sphere contains the sphere of center $\xi$ and radius $r(\xi)$, it is clear that the collection $\mathcal{C}$ formed by adding the new spheres to the collection $\mathcal{C}_{1}$ has the property that any two of its members intersect, but the intersection of the whole $\mathcal{C}$ is void. Every point $x \in \mathbb{E}$ is then the center of one and just one member of $\mathcal{C}$ : call $r(x)$ its radius. Then we have the inequality (7) satisfied for all $x, y \in \mathbb{E}$. Moreover for any given $\xi \in \mathbb{E}$, the inequality $r(x) \geqq\|x-\xi\|$ cannot be valid for all $x \in \mathcal{F}$, for if it could, $\xi$ would be common to all members of $\mathcal{C}$. Now apply Lemma 2 and consider a real function $\rho$ on $\&$ satisfying conditions (I), (II), and (III) and such that $r \geqq \rho$. It is then clear that, for any given $\xi \in \mathbb{E}$, the inequality $\rho(x) \geqq\|x-\xi\|$ cannot be valid for all $x \in \mathbb{E}$. This implies, in particular, that $\rho$ satisfies also condition (IV), for $\rho(\xi) \leqq 0$ for some $\xi \in$ \& would imply

$$
\rho(x) \geqq \rho(x)+\rho(\xi) \geqq\|x-\xi\|,
$$


which is impossible.

Having this function $\rho$, we can apply Lemma 1 and obtain an immediate extension $\mathfrak{X}$ of $\&$ containing a point $\zeta$ not in $\&$ such that $\rho(x)=\|x-\zeta\|$ for all $x \in \mathbb{E}$. We say that there is no projection $P$ of $\mathfrak{X}$ onto $\mathbb{E}$ with norm equal to 1. For otherwise $\zeta$ would have an image $\xi=P(\zeta) \in \Subset$ and we would have

$$
\|P(y)-\xi\|=\|P\{P(y)-\zeta\}\| \leqq\|P(y)-\zeta\|
$$

for any $y \in \mathfrak{X}$ : since $P(y)=x$ is an arbitrary point of $\mathbb{E}$, this would mean that $\|x-\xi\| \leqq \rho(x)$ for all $x \in \mathcal{E}$, which is impossible.

Consider then the vector subspace $\mathfrak{S}=\mathbb{E}$ of $\mathfrak{X}$ and the identity mapping $f: \Im \rightarrow \mathbb{E}$. Then $\|f\|=1$, but there is no linear extension $F: \mathfrak{X} \rightarrow \mathbb{E}$ such that $\|F\|=1$, for such an extension would be a projection of $\mathfrak{X}$ onto $\mathbb{E}$ with norm equal to 1 .

The proof of Theorem 1 is therefore completed.

4. Structure of normed spaces whose collections of spheres have the binary intersection property. The binary intersection property for the collection of spheres of a normed space is a very strong one, as one can see by remarking that any real Hilbert space of dimension not less than 2 does not have this property. This fact, however, does not prevent the existence of a large number of normed spaces of any dimension with that property. In this and in the next section, we shall investigate the structure of normed spaces enjoying the binary intersection property.

Suppose that we have a vector space $₹$ which is ordered in such a way as to become a complete vector lattice, that is:

(1) $F$ is an ordered vector space: this means that $x \leqq y$ implies $x+z \leqq y+z$ and $\lambda x \leqq \lambda y$ for any $x, y, z \in \mathbb{E}$ and any scalar $\lambda \geqq 0$,

(2) $₹$ is a complete lattice.

We shall also assume that:

(3) (E has an order unity $e$ : this is an element $e>0$ such that, given any $x \in \mathbb{F}$ we have $-\lambda e \leqq x \leqq \lambda e$ for some scalar $\lambda$.

Consider a fixed order unity $e$. For each $x \in \&$, call $\|x\|$ the greatest lower bound of all scalars $\lambda$ for which $-\lambda e \leqq x \leqq \lambda e$. It is not hard to verify, and in fact it is well known, that $\|x\|$ is a norm function on the vector space $\leftleftarrows$ and that the sphere of center $\xi$ and radius $r \geqq 0$ is identical to the segment $[\xi-r e, \xi+r e]$. Therefore every order relation on $\&$ making $\&$ into a complete vector lattice with an order unity and every choice of this unity allow us to make $\&$ into a normed space in a natural way.

We say that the collection of spheres of $\&$ has the binary intersection property. Since every sphere is a segment, it will be sufficient to prove that the more inclusive collection of segments of $\&$ has the binary intersection property. This follows from one-half of the lemma below. Later we shall need the other half.

LemMA 3. An ordered set $\&$ is a complete lattice if and only if it has the in- 
terpolatory property and the collection of its segments has the binary intersection property.

In fact, if $\mathbb{E}$ is complete and $x_{1}, x_{2} \leqq y_{1}, y_{2}$, then $x_{1}$ and $x_{2}$ are bounded from above and so $x_{1} \vee x_{2}$ has a meaning; similarly, $y_{1} \wedge y_{2}$ has a meaning and we have $x_{1} \bigvee x_{2} \leqq y_{1} \wedge y_{2}$. Then if $z$ satisfies $x_{1} \bigvee x_{2} \leqq z \leqq y_{1} \wedge y_{2}$, we shall have $x_{1}, x_{2} \leqq z \leqq y_{1}, y_{2}$ showing that $\&$ has the interpolatory property. Consider now any nonempty collection of segments $\left[x_{\lambda}, y_{\lambda}\right]$ any two of which intersect, that is, for each pair $\alpha, \beta$ there exists some $z_{\alpha \beta}$ such that $x_{\alpha}, x_{\beta} \leqq z_{\alpha \beta} \leqq y_{\alpha}, y_{\beta}$. This implies $x_{\alpha} \leqq y_{\beta}$ for any $\alpha, \beta$. In particular, the $x_{\alpha}$ are bounded from above and so $\bigvee_{\alpha} x_{\alpha}$ has a meaning; similarly, $\Lambda_{\beta} y_{\beta}$ has a meaning and we have $\bigvee_{\alpha} x_{\alpha} \leqq \Lambda_{\beta} y_{\beta}$. Take any $z$ for which $\bigvee_{\alpha} x_{\alpha} \leqq z \leqq \Lambda_{\beta} y_{\beta}$. Then $x_{\alpha} \leqq z \leqq y_{\beta}$ for any $\alpha$, $\beta$ and in particular for $\alpha=\beta=\lambda$, that is, $z \in\left[x_{\lambda}, y_{\lambda}\right]$ for any $\lambda$, showing that the collection of segments of $\&$ has the binary intersection property.

Conversely, assume that $₹$ satisfies the two conditions of the lemma. Let $\left\{x_{\lambda}\right\}$ be a nonempty collection of points of $\&$ bounded from above. Call $\left\{y_{\mu}\right\}$ the collection of upper bounds of $\left\{x_{\lambda}\right\}$. Consider the collection of segments $\left[x_{\lambda}, y_{\mu}\right]$. From the interpolatory property it follows that any two of these segments have a point in common. From the binary intersection property it follows then that these segments have a point $z$ in common, that is, $x_{\lambda} \leqq z \leqq y_{\mu}$ for any $\lambda, \mu$ : the first half means that $z$ is an upper bound of $\left\{x_{\lambda}\right\}$, and the second half that $z$ is the least upper bound, as desired.

The order unity $e$ is an extreme point of the unity sphere $U$ of $₹$. In fact, assume that $x$ and $y$ belong to $U, x \neq e, y \neq e$. Then $e$ does not belong to the segment $[x, y]$ : in fact, we have $-e \leqq x \leqq e,-e \leqq y \leqq e$ and therefore $x<e, y<e$. From this it follows that $\lambda x+(1-\lambda) y<\lambda e+(1-\lambda) e=e$ and a fortiori $\lambda x$ $+(1-\lambda) y \neq e$ for any $\lambda, 0 \leqq \lambda \leqq 1$, showing that $e$ is an extreme point of $U$.

Finally we say that the knowledge of the order unity $e$ together with the norm function on $\&$ determine uniquely the order relation on $\mathbb{E}$. It is equivalent to determine the set of all positive elements. We say that $x \geqq 0$ if and only if it can be written as $x=\lambda(u+e)$, where $\lambda \geqq 0$ and $\|u\| \leqq 1$. In fact, if $x$ has this form, then $-e \leqq u \leqq e$ and so $u+e \geqq 0$ from which $x \geqq 0$. Conversely, if $x>0$ (the case $x=0$ being trivial), we take $\lambda \geqq\|x\| / 2$, from which $\|x\| \leqq 2 \lambda$ and $0 \leqq x \leqq 2 \lambda e$ or equivalently $-e \leqq(x-\lambda e) / \lambda \leqq e$; now if we define $u$ $=(x-\lambda e) / \lambda$ we obtain the desired decomposition of $x$.

Conversely, suppose that we have a normed space $\&$ and an extreme point $e$ of its unity sphere $U$. If the collection of spheres of $\&$ has the binary intersection property, then there exists one and only one order relation on $\&$ with respect to which $\&$ is a complete vector lattice having $e$ as order unity such that the norm deduced from the order relation and the order unity is precisely the norm already given in $\&$. Since the uniqueness of the order relation has just been proved, it remains only to prove its existence.

Call $P$ the set of all elements $x \in \mathbb{E}$ of the form $x=\lambda(u+e)$, where $\lambda \geqq 0$ 
and $\|u\| \leqq 1\left(^{6}\right)$. It is clear that $0 \in P$ and that $\lambda P \subset P$ for any $\lambda \geqq 0$. Moreover $P+P \subset P$. In fact, if $x=\lambda(u+e), y=\mu(v+e)$ with $\lambda \geqq 0,\|u\| \leqq 1, \mu \geqq 0$ and $\|v\| \leqq 1$, then $x+y=\nu(w+e)$, where $w=(\lambda u+\mu v) /(\lambda+\mu)$ and $\nu=\lambda+\mu$ (we have excluded the trivial case $\lambda=\mu=0)$. But $\nu \geqq 0$ and $\|w\| \leqq 1$ and therefore $x+y \in P$. Finally $P \cap(-P)=0$. In fact, assume that $x=\lambda(u+e),-x=\mu(v+e)$ with $\lambda \geqq 0,\|u\| \leqq 1, \mu \geqq 0$ and $\|v\| \leqq 1$. Then $(\lambda+\mu) e+\lambda u+\mu v=0$. If $\lambda+\mu=0$, that is, $\lambda=\mu=0$, then we get $x=0$. If $\lambda+\mu>0$, then $-e=(\lambda u+\lambda v) /(\lambda+\mu)$ together with the fact that $-e$ is also an extreme point of the unity sphere imply either $-e=u$ or $-e=v$, and in any case we have $x=0$. Therefore $P$ has all properties that are needed in order that $\&$ be an ordered vector space under the definition $x \leqq y \rightleftarrows y-x \in P$, with $P$ as the set of all positive elements.

We have the relation

$$
U=(e-P) \cap(-e+P) .
$$

In fact, if $u \in U$, then $u=e-1(e-u) \in e-P$ and $u=-e+1(u+e) \in-e+P$, proving that relation $(8)$ is valid with $=$ replaced by $C$. To prove the inclusion in the reversed sense, consider $u \in(e-P) \cap(-e+P)$. Then $u=e-\alpha(v+e)$ and $u=-e+\beta(w+e)$, where $\alpha \geqq 0,\|v\| \leqq 1, \beta \geqq 0$ and $\|v\| \leqq 1$. From the first expression for $u$ we get $u=(1-\alpha) e+\alpha(-v)$ : since $e$ and $-v$ belong to $U$, the relation $\alpha \leqq 1$ would imply $u \in U$ as desired; therefore let us assume that $\alpha>1$. Similarly $u=(1-\beta)(-e)+\beta w$ and the fact that $-e$ and $w$ belong to $U$ show that $\beta \leqq 1$ would imply $u \in U$ as desired; therefore we shall assume that $\beta>1$.

Let us submit $U$ to the translation taking - $e$ into 0 , next to the similitude of positive ratio $\alpha-1$ and center at the origin, and finally to the translation taking 0 into $u$. The final result $A=u+(\alpha-1)(e+U)$ will be the sphere with center $u+(\alpha-1) e$ and radius $\alpha-1$. We have

$$
A \subset u+(\alpha-1) P=u+P
$$

because $U \subset-e+P$. Moreover $u=(1-\alpha) e+\alpha(-v)$ implies that $-v=u$ $+(\alpha-1)(e+v) \in A$ and so $A$ and $U$ have the point $-v$ in common. Let us start again and submit $U$ to the translation taking $e$ into 0 , next to the similitude of positive ratio $\beta-1$ and center at the origin, and finally to the translation taking 0 into $u$. The final result $B=u+(\beta-1)(-e+U)$ is the sphere with center $u+(\beta-1)(-e)$ and radius $\beta-1$. We have

$$
B \subset u+(\beta-1)(-P)=u-P
$$

because $U \subset e-P$. Noticing that $u=(1-\beta)(-e)+\beta w$ implies $w=u$ $+(\beta-1)(-e-w) \in B$ we see that $B$ and $U$ have the point $w$ in common. On the other hand $u=u+(\alpha-1)(e-e) \in A$ and $u=u+(\beta-1)(-e+e) \in B$ show that $A$ and $B$ have the point $u$ in common. By the binary intersection

(6) The idea we use here of determining an order relation in a normed space from an extreme point is known. 
property of the collection of spheres, we see that $A \cap B \cap U$ is not empty. Now from the relations (9) and (10) we see that

$$
A \cap B \subset(u+P) \cap(u-P) \text {. }
$$

Moreover the relation $P \cap(-P)=0$ submitted to the translation taking 0 into $u$ gives

$$
(u+P) \cap(u-P)=u .
$$

This shows that $A \cap B$ is reduced to the point $u$ and this implies that $u \in U$. The proof of the equality (8) is therefore finished.

Every closed sphere is identical to a segment. First of all we prove that $U$ is identical to $[-e, e]$. In fact, $x \in[-e, e]$ if and only if $-e \leqq x \leqq e$, that is, $e \pm x \geqq 0$ which is equivalent to $e \pm x \in P$ or to $x \in \pm(e-P):$ this shows that

$$
[-e, e]=(e-P) \cap(-e+P)
$$

and it is then sufficient to apply relation (8). From this it follows readily that the sphere of center $\xi$ and radius $r$ is identical to the segment $[\xi-r e, \xi+r e]$.

Every segment in $\$$ can be expressed as an intersection of two spheres. In fact, consider the segment $[x, y]$ and set

$$
\begin{gathered}
r=\|y-x\| / 2, \quad \bar{x}=y-2 r e, \quad \bar{y}=x+2 r e, \\
m=(\bar{x}+y) / 2, \quad n=(x+\bar{y}) / 2 .
\end{gathered}
$$

Consider the spheres $M$ and $N$ of centers $m$ and $n$, respectively, and radii equal to $r$. Then

$$
M=[m-r e, m+r e]=[\bar{x}, y], \quad N=[n-r e, n+r e]=[x, \bar{y}] .
$$

Since $\|y-x\|=2 r$, we have $y-x \leqq 2 r e$ and so $\bar{x} \leqq x$ and $y \leqq \bar{y}$. From this it follows that $M \cap N=[x, y]$ for $x \leqq t \leqq y$ is obviously equivalent to $\bar{x} \leqq t \leqq y$ plus $x \leqq t \leqq \bar{y}$.

The collection of segments of the ordered set $(5$ has the binary intersection property. In fact, let $\left\{S_{\lambda}\right\}$ be any nonempty collection of segments of $\&$, any two of which intersect. Write $S_{\lambda}=M_{\lambda} \cap N_{\lambda}$, where $M_{\lambda}$ and $N_{\lambda}$ are spheres. Then the collection of spheres formed by all $M_{\lambda}$ and all $N_{\lambda}$ has the property that any two of them intersect; this follows simply from the relation:

$$
M_{\alpha} \cap N_{\alpha} \cap M_{\beta} \cap N_{\beta}=S_{\alpha} \cap S_{\beta} .
$$

Therefore there is a point common to all these spheres, and this point will clearly be common to all segments $S_{\lambda}$.

The ordered set $₹$ has the interpolatory property. In fact, let $x_{1}, x_{2}, y_{1}, y_{2} \in \notin$ be given with $x_{1}, x_{2} \leqq y_{1}, y_{2}$. Consider the segment $\left[x_{i}, y_{i}\right]$ and write it as the intersection of two spheres: $\left[x_{i}, y_{i}\right]=M_{i} \cap N_{i}$, where

$$
M_{i}=\left[\bar{x}_{i}, y_{i}\right], \quad N_{i}=\left[x_{i}, \bar{y}_{i}\right],
$$




$$
\bar{x}_{i}=y_{i}-2 r_{i} e, \quad \bar{y}_{i}=x_{i}+2 r_{i} e, \quad r_{i}=\left\|y_{i}-x_{i}\right\| / 2 .
$$

We say that any two of the spheres $M_{1}, N_{1}, M_{2}$, and $N_{2}$ do intersect. This is obvious for the pair $M_{1}, N_{1}$ and for the pair $M_{2}, N_{2}$. Let us prove it for the pair $M_{1}, M_{2}$. We have to show only that the distance of the centers of $M_{1}$ and $M_{2}$ is not greater than the sum of their radii, that is:

$$
\left\|\frac{\bar{x}_{1}+y_{1}}{2}-\frac{\bar{x}_{2}+y_{2}}{2}\right\| \leqq r_{1}+r_{2} .
$$

Replacing $\bar{x}_{1}$ and $\bar{x}_{2}$ by their expressions, we get the equivalent condition

$$
\left\|y_{1}-y_{2}+\left(r_{2}-r_{1}\right) e\right\| r_{1}+r_{2},
$$

which amounts to

$$
-2 r_{2} e \leqq y_{1}-y_{2} \leqq 2 r_{1} e .
$$

But

$$
y_{1}-y_{2}=\left(y_{1}-x_{1}\right)+\left(x_{1}-y_{2}\right) \leqq y_{1}-x_{1} \leqq 2 r_{1} e
$$

because $\left\|y_{1}-x_{1}\right\|=2 r_{1}$. Also

$$
y_{1}-y_{2}=\left(y_{1}-x_{2}\right)+\left(x_{2}-y_{2}\right) \geqq x_{2}-y_{2} \geqq-2 r_{2} e
$$

because $\left\|y_{2}-x_{2}\right\|=2 r_{2}$. We have thus proved that $M_{1}$ and $M_{2}$ do intersect. By using the same type of reasoning, we complete the proof that any two of those four spheres do intersect. By the binary intersection property of the collection of spheres, there is a point $z$ common to these spheres: then $z$ is in $M_{1} \cap N_{1}$ and also in $M_{2} \cap N_{2}$, showing that $x_{1}, x_{2} \leqq z \leqq y_{1}, y_{2}$ as desired.

(5) is a complete lattice. This follows readily from the two lastly proved properties and from Lemma 3.

$e$ is an order unity for E. In fact, we have clearly $e>0$. Moreover, given any $x$, it is contained in the sphere of center 0 and radius $\lambda \geqq\|x\|$. Since this sphere is identical to the segment $[-\lambda e, \lambda e]$, we have $-\lambda e \leqq x \leqq \lambda e$.

The norm deduced from the order relation on $\&$ and the order unity $e$ is identical to the original norm. This follows simply from the fact that both norms have the same unity sphere, namely the segment $[-e, e]$.

We have thus completed the proof of the following theorem:

THEOREM 2. If \& is a complete vector lattice with an order unity e, then $($, endowed with the norm deduced from its order relation and e, becomes a normed space whose collection of spheres has the binary intersection property and whose unity sphere has e as an extreme point. Conversely, given any normed space $₹$ whose collection of spheres has the binary intersection property and whose unity sphere contains $e$ as an extreme point, there is one and only one way of making (5 into a complete vector lattice having $e$ as an order unity such that the norm deduced from the order relation and $e$ is identical to the given norm. 
As we have stated in the introduction, we do not know whether the unity sphere of every normed space, whose collection of spheres has the binary intersection property, must contain an extreme point. It seems that this is likely to be the case. In this situation we cannot apply the standard theorem of Krein and Milman about the existence of extreme points $\left({ }^{7}\right)$ because the unity sphere is not weakly compact, except in the trivial case where $(5$ is finite-dimensional (see next section). However, the binary intersection property for the collection of spheres of a normed space has a certain compactness character. In any case, Theorem 2 gives us a first information about the structure of a normed space whose collection of spheres has the binary intersection property, and satisfying the additional condition (that may be redundant) that its unity sphere contains an extreme point.

Let us now examine the finite-dimensional case in which a simple result is to be expected. Call $\Re^{n}$ the $n$-dimensional euclidean space considered as a vector space in the usual way, ordered component by component and normed by

$$
\|x\|=\left|x_{1}\right| \vee \cdots \vee\left|x_{n}\right| \text { if } x=\left\{x_{1}, \cdots, x_{n}\right\}
$$

This is the norm that we have to consider on $\Re^{n}$ when we think of it as the normed space of all (continuous) functions over the (compact) space $\{1,2, \cdots, n\}$. It is not hard to see that the collection of spheres of $\Re^{n}$ has the binary intersection property: this follows from the existence of the order unity $e=\{1,1, \cdots, 1\}$. Therefore every normed space that is isomorphic to $\Re^{n}$ has dimension $n$ and its collection of spheres has the binary intersection property.

Conversely, if a normed space $\mathbb{E}$, whose collection of spheres has the binary intersection property, is of finite dimension $n$, then its unity sphere contains an extreme point $e$. We can then apply Theorem 2 and set up an order relation on $\mathbb{E}$ as was described before. Applying a known theorem $\left({ }^{8}\right)$, we can say that the order relation on $\&$ has a component character, that is, there exists a base $e_{1}, e_{2}, \cdots, e_{n}$ for $\left(5\right.$ such that $x=\sum_{1}^{n} x_{i} e_{i} \geqq 0$ if and only if $x_{i} \geqq 0(i=1,2, \cdots, n)$. We can assume, of course, that $\left\|e_{i}\right\|=1$. Then we assert that $e=\sum_{1}^{n} e_{i}$. In fact, write $e=\sum_{1}^{n} \lambda_{i} e_{i}$. Since $e \geqq 0$, we have all $\lambda_{i} \geqq 0$. Moreover $\left\|e_{1}\right\|=1$ implies $e_{1} \leqq e=\sum_{1}^{n} \lambda_{i} e_{i}$ and therefore $1 \leqq \lambda_{1}$. On the other hand $\left\|e_{1}\right\|=1$ and $e_{1} \geqq 0$ imply that $e_{1} \leqq \theta e=\sum_{1}^{n} \theta \lambda_{i} e_{i}$ is false for any $\theta, 0<\theta<1$ : this requires that $\theta \lambda_{1}<1$ and making $\theta \rightarrow 1$ we see that $\lambda_{1} \leqq 1$. Therefore $\lambda_{1}=1$ and similarly for all $\lambda_{i}$. Having proved this, we see that $x=\sum_{1}^{n} x_{i} e_{i}$ satisfies $-\lambda e \leqq x \leqq \lambda e$ if and only if $-\lambda \leqq x_{i} \leqq \lambda$ which amounts to $\lambda \geqq\left|x_{i}\right|$ for $i=1,2, \cdots, n$. Therefore $\|x\|=\left|x_{1}\right| \vee \cdots \vee\left|x_{n}\right|$. This shows that the mapping $x \rightarrow\left\{x_{1}, \cdots, x_{n}\right\}$ is not only a vector space isomorphism between

(7) See Krein and Milman [9] or Godement [6].

(8) See Youdine [16] and Nagy [11]. 
$\leftleftarrows$ and $\Re^{n}$, but it is also an isometry. Therefore we have proved the following theorem $\left({ }^{9}\right)$.

THEOREM 3. The collection of spheres of a normed space \& of dimension $n$ has the binary intersection property if and only if $\mathbb{E}$ is isomorphic in the norm and vector sense to the vector space $\Re^{n}$ normed by (11).

The above theorem shows, in particular, that in the finite-dimensional case the unity sphere of a normed space whose collection of spheres has the binary intersection property looks like a cube.

Finally, let us remark that if a normed space is actually given as a complete vector lattice with an order unity related to the norm as described before, then the proof that it has the extension property can be carried out exactly as is given in Banach's book.

5. Relation to complete Boolean algebras. Suppose that we have a normed space $f$ whose collection of spheres has the binary intersection property and whose unity sphere has $e$ as an extreme point. Then we can make \& into a complete vector lattice with $e$ as order unity as was described before. It is not hard to verify that $\&$ is then an $(M)$-space in the sense of Kaku$\operatorname{tani}\left({ }^{10}\right)$. Therefore there is a compact Hausdorff space $K$ whose normed lattice $\widehat{S}_{K}$ of real continuous functions is isomorphic in the vector, norm, and order sense to $\&$ in such a way that $e$ is mapped into the unity function. We can also say, by the Banach-Stone theorem $\left({ }^{11}\right)$ that $K$ is unique up to homeomorphisms, even if we know only that $\xi_{K}$ is isomorphic in the norm and vector sense (but not necessarily in the order sense) to $(5$.

The space $K$ has however to be more than a simple compact Hausdorff space because its lattice of real continuous functions, being isomorphic to $\&$, has to be complete. We shall need at this moment the following definition.

A topological space will be said to be extremally disconnected if it is a completely regular space in which the closure of every open set is open. It is not hard to see that the lattice of all real continuous functions over a completely regular space is complete if and only if it is extremally disconnected. Since the unity function on $K$ is an order unity for $\mathfrak{S}_{K}$ when $K$ is compact, we have the following theorem.

THEOREM 4. Every normed space whose collection of spheres has the binary intersection property and whose unity sphere contains an extreme point is isomorphic in the vector and norm sense to the normed space of all real continuous functions over an extremally disconnected compact Hausdorff space, which is unique up to homeomorphisms. Conversely, if a normed space is isomorphic in

( $\left.{ }^{9}\right)$ A more general proposition is as follows: let $C$ be a given compact set with nonempty interior in a finite-dimensional real vector space. Then the collection of all translates of $C$ has the binary intersection property if and only if $C$ is a cube.

(10) See Kakutani [7] and also Krein and Krein [8].

(11) See, for instance, Arens and Kelley [1]. 
the vector and norm sense to the normed space of all real continuous functions over an extremally disconnected compact Hausdorff space, then its collection of spheres has the binary intersection property.

From the above theorem and known results, we can deduce the following theorem.

THEOREM 5. The unity sphere of a normed space whose collection of spheres has the binary intersection property is weakly compact if and only if the space is finite-dimensional $\left({ }^{12}\right)$.

In fact, if the unity sphere is weakly compact, we can apply the theorem of Krein and Milman $\left(^{7}\right)$ and say that it contains some extreme point. Then by the previous theorem $\&$ is isomorphic to the normed space of all real continuous functions over a compact Hausdorff space: but it is known that the unity sphere of such a space is weakly compact if and only if $K$ is a finite set $\left({ }^{13}\right)$. Therefore $\&$ must be finite-dimensional. The converse is of course trivial.

Theorem 4 says that, roughly speaking, there are as many normed spaces whose collections of spheres have the binary intersection property and whose unity spheres contain extreme points as there are extremally disconnected compact Hausdorff spaces. However the extremally disconnected compact Hausdorff spaces are to be considered as highly pathological, and they must be used only as auxiliary tools. It seems therefore convenient to replace them by a perfectly equivalent notion, that of a complete Boolean algebra, which has no pathological aspect.

Suppose that we have an extremally disconnected compact Hausdorff space $K$. Let $B$ be the collection of all closed and open subsets of $K$. It is not hard to verify that $B$ is a complete Boolean algebra, the supremum in $B$ of any collection of elements of $\mathcal{B}$ being the closure of their point set union. Since every extremally disconnected space is totally disconnected, we can say $\left({ }^{14}\right)$ that the knowledge of $B$ up to an isomorphism determines the knowledge of $K$ up to a homeomorphism. Conversely, given any complete Boolean algebra $B$, by virtue of Stone's theorem it is isomorphic as a Boolean algebra to the Boolean algebra of all open and closed sets of a totally disconnected compact Hausdorff space $K$ : it is not hard to see that from the completeness of $B$ we can infer the extra property that $K$ is extremally disconnected $\left.{ }^{15}\right)$. Therefore via Stone's theorem there is a natural one-to-one correspondence between extremally disconnected compact Hausdorff spaces

('2) In other words (see Dieudonne [5]), the space is reflexive if and only if it is finitedimensional.

${ }^{(13)}$ We use here the known fact that the unity sphere of the normed space of all real bounded continuous functions over a completely regular space is weakly compact if and only if this space is finite.

(14) See Stone [13].

(1) See Stone [14], [15] for more complete results in this connection. 
and complete Boolean algebras, of course up to the corresponding admissible equivalences.

It is not hard to see that a function is an extreme point of the unity sphere of the normed space of all real continuous functions over a compact Hausdorff space if and only if it takes on the space only the values 1 and -1 . Therefore there exists a natural one-to-one correspondence between the extreme points of this sphere and the open and closed subsets of the space. All that we have said leads to the following theorem.

THEOREM 6. Let \& be a normed space whose collection of spheres has the binary intersection property and let $e$ be an extreme point of its unity sphere $U$. If we make (5 into a complete vector lattice with $e$ as order unity and the segment $[-e, e]$ as the unity sphere, then the collection $\mathcal{E}$ of all extreme points of $U$ endowed with the induced ordering relation will be a complete Boolean algebra with $e$ as the last element. Different choices of e make $\mathcal{E}$ into different but isomorphic complete Boolean algebras. Conversely, given any complete Boolean algebra, there is a normed space, unique up to an isomorphism, whose collection of spheres has the binary intersection property and whose collection of extreme points is not empty and is a complete Boolean algebra (for any choice of a particular extreme point) isomorphic to the given one.

Roughly speaking we can then say that there are as many normed spaces whose collections of spheres have the binary intersection property and whose unity spheres contain extreme points as there are complete Boolean algebras.

We finish this section by stating the following as yet unsolved problems:

(1) Does the unity sphere of a normed space whose collection of spheres has the binary intersection property have at least one extreme point? By virtue of Theorem 4, the existence of at least one extreme point is equivalent to the fact that the convex hull of the set of all extreme points is dense in the unity sphere.

(2) Suppose that we have a Banach space $\&$ with the following properties:

(a) If we have any finite collection of spheres any two of which intersect, then the collection has a nonvoid intersection.

(b) There is an extreme point in the unity sphere.

Is this normed space isomorphic to the normed space of all continuous functions over a compact Hausdorff space?

If $\&$ is finite-dimensional, then the answer is yes: in fact, in this case every sphere is compact and from the property (b) above it follows easily that the collection of spheres has the binary intersection property. It remains only to apply Theorem 3 .

In this case it is easy to see that (a) does not imply (b): it is sufficient to consider the normed space of all real continuous functions over a compact Hausdorff space vanishing at a nonisolated point. It is also easy to see that property (a) does imply the completeness of a normed space: it is sufficient to 
consider the normed space of real functions that have a derivative on the unity segment, the norm of each function being the least upper bound of its absolute value.

\section{BIBLIOGRAPHY}

1. R. F. Arens and J. L. Kelley, Characterizations of the space of continuous functions over a compact Hausdorff space, Trans. Amer. Math. Soc. vol. 62 (1947) pp. 499-508.

2. S. Banach, Théorie des opérations linéaires, Warsaw, 1932.

3. I. S. Cohen, On non-Archimedean normed spaces, Indagationes Mathematicae vol. 10 (1948) pp. 244-249.

4. J. Dieudonné, Sur le thêorème de Hahn-Banach, Revue Scientifique vol. 79 (1941) pp. 642-643.

5. - La dualité dans les espaces vectoriels topologiques, Ann. École Norm. vol. 59 (1942) pp. 107-139.

6. R. Godement, Les fonctions de type positif et la thêorie des groupes, Trans. Amer. Math. Soc. vol. 63 (1948) pp. 1-84.

7. S. Kakutani, Concrete representations of abstract (M)-spaces, Ann. of Math. vol. 42 (1941) pp. 994-1024.

8. M. Krein and S. Krein, Sur l'espace des fonctions continues definies sur un bicompact de Hausdorff et ses sousespaces semiordonnés, Rec. Math. (Mat. Sbornik) N.S. vol. 13 (1943) pp. $1-37$.

9. M. Krein and D. Milman, On the extreme points of regularly convex sets, Studia Mathematica vol. 9 (1940) pp. 133-138.

10. S. Lefschetz, Algebraic topology, Amer. Math. Soc. Colloquium Publications, vol. 27, New York, 1942.

11. B. de Sz. Nagy, Sur les lattis linéaires de dimension finie, Comment. Math. Helv. vol.17 (1945) pp. 209-213.

12. F. Riesz, Sur quelques notions fondamentales dans la theorie générale des opérations linéaires, Ann. of Math. vol. 41 (1940) pp. 174-206.

13. M. H. Stone, Applications of the theory of Boolean rings to general topology, Trans. Amer. Math. Soc. vol. 41 (1937) pp. 375-481.

14. - A general theory of spectra, I and II, Proc. Nat. Acad. Sci. U.S.A. vol. 26 (1940) pp. 280-283, vol. 27 (1941) pp. 83-87.

15. - Boundedness properties in function-lattices, Canadian Journal of Mathematics vol. 1 (1949) pp. 176-186.

16. A. Youdine, Solution de deux problèmes de la théorie des espaces semiordonnes, C. R. (Doklady) Acad. Sci. URSS. vol. 27 (1939) pp. 418-422.

The University of Chicago, Chicago, Ill. 